INPLASY

PROTOCOL

To cite: Zhao et al. Association between IncRNA GAS5 polymorphism rs145204276 and the risk of cancer. Inplasy protocol 202170036. doi: 10.37766/inplasy2021.7.0036

Received: 12 July 2021

Published: 12 July 2021

Corresponding author: Lanhua Tang

orchidtang@126.com

Author Affiliation:

Central South University.

Support: National Natural

Science Found.

Review Stage at time of this submission: completed but not published.

Conflicts of interest:

None declared.

\section{Association between IncRNA GAS5 polymorphism rs145204276 and the risk of cancer}

Zhao, SS1; Liu, P2; Ruan, Z3; Li, JH4; Zeng, S5; Zhong, MZ6; Tang, $\mathrm{LH}^{7}$.

Review question / Objective: GAS5 plays important roles in various tumors, and an increasing number of studies have explored the association of GAS5 polymorphism rs 145204276 and cancer risk; however, the results are inconclusive. So we conducted this meta-analysis and systematic review.

Eligibility criteria: (1) a clinical case-control study; (2) focus on the association between IncRNA SNPs and cancer risk; (3) the distribution of genotype in controls was consistent with the Hardy-Wein-berg equilibrium (HWE).

INPLASY registration number: This protocol was registered with the International Platform of Registered Systematic Review and Meta-Analysis Protocols (INPLASY) on 12 July 2021 and was last updated on 12 July 2021 (registration number INPLASY202170036).

\section{INTRODUCTION}

Review question / Objective: GAS5 plays important roles in various tumors, and an increasing number of studies have explored the association of GAS5 polymorphism rs145204276 and cancer risk; however, the results are inconclusive.
So we conducted this meta-analysis and systematic review.

Condition being studied: Long non-coding RNAs (IncRNAs) are a group of non-coding functional RNAs, whose length are more than 200 nucleotides. As the development of high throughput sequencing technology, 
IncRNAs are known as functional RNAs that play important roles in cell cycles, apoptosis, epigenetics, and regulation of gene expression. GAS5 is identified as a tumor suppressor gene in various kinds of tumors, including breast cancer, gastric cancer, bladder cancer and so on. GAS5 is significantly reduced in breast cancer samples relative to adjacent normal breast epithelial tissues, and its expression induces growth arrest and apoptosis of breast cancer cell lines. GAS5's expression in gastric cancer is also lower compared with the normal counterparts, and it enhances G1 cell cycle arrest via the YBX1/ p21 pathway. So the GAS5 plays important roles in various tumors. Single nucleotide polymorphism (SNP) is one of the main genetic variations, accounting for more than $90 \%$ of all known polymorphisms]. Recent study shows polymorphisms in the promoter region of IncRNAs can regulate the mRNA expression level thus the protein expression level. There is a GAS5 5-bp insertion/deletion (indel) polymorphism (rs145204276, AGGCA/-) in the GAS5 promoter region, and allele del increases luciferase activity and expression levels of GAS5. Aminian et al found that del/del genotype showed protective effects on gastric cancer (GC) risk through modulating protein Cyclin-dependent kinase inhibitor 1B (p27Kip1) expression. Li $Q$ et al also found that allele del was associated with decreased risk of GC, lymph node metastasis and distant metastasis of GC, and also with a higher survival rate. These characteristics of GAS5 rs145204276 were also observed in the colorectal cancer (CRC), lung cancer, breast cancer, osteosarcoma. However, other research groups found that rs145204276 del allele increased the risk of hepatocellular carcinoma (HCC)and glioma. These controversies required further analysis on rs145204276, so we conduct this meta- analysis to evaluate the role of rs145204276 in various tumor.

\section{METHODS}

Search strategy: A literature search of PubMed, Medline, EmBase, and Cochrane library from the inception of each database was performed, using the following keywords: "GAS5/ growth arrest-specific 5", "polymorphisms /SNP /single nucleotide polymorphism", and "cancer/ carcinoma/tumor/neoplasm". All selected studies met the following criteria: (1) a clinical case-control study; (2) focus on the association between IncRNA SNPs and cancer risk; (3) the distribution of genotype in controls was consistent with the HardyWein-berg equilibrium (HWE). (4) published in English language; The exclusion criteria were: (1) duplicate studies; (2) not relevant to cancer or IncRNA SNPs; or (3) no available data and the authors could not be contacted.

Participant or population: Included association studies met three criteria: they must 1) evaluated associations between GAS5 rs145204276 polymorphisms and the susceptibility to develop cancers in diverse populations, 2) used a case-control design, and 3 ) included genotypic frequencies.

Intervention: Polymorphism of GAS rs145204276 in various cancers patients.

Comparator: Controls with no history of diseases.

Study designs to be included: Case-control studies.

Eligibility criteria: (1) a clinical case-control study; (2) focus on the association between IncRNA SNPs and cancer risk; (3) the distribution of genotype in controls was consistent with the Hardy-Wein-berg equilibrium (HWE).

Information sources: PubMed, Medline, EmBase, and Cochrane library.

Main outcome(s): Risk of various cancers.

Data management: Titles and abstracts were independently evaluated for relevance by two reviewers (SS Zhao and LH Tang). Full manuscripts of individual studies were then thoroughly reviewed according to the inclusion and exclusion criteria. 
Quality assessment / Risk of bias analysis: Newcastle-Ottawa scale will be used to appraise the included studies.

Strategy of data synthesis: PRISMA guidelines will be used and applied. Appraisal of selected studies using the Newcastle-Ottawa scale. A meta-analysis of ORs (pooling OR) with heterogeneity verification, publication bias, etc.

Subgroup analysis: As to genotype comparison, the risk of six genetic models including the dominant model, recessive model, additive model, homozygous model, heterozygous model, and allele model was estimated respectively. Subgroup analysis was performed by type of cancer and ethnicity.

Sensitivity analysis: Sensitivity analyses were conducted by omitting a single study in each.

Language: English.

Country(ies) involved: China.

Keywords: GAS5, LncRNA, polymorphism, SNPs, case-control, cancer, meta-analysis.

Dissemination plans: Publication in an international peer-reviewed and Medlineindexed journal.

Contributions of each author:

Author 1 - Shushan Zhao.

Author 2 - Ping Liu.

Author 3 - Zhe Ruan.

Author 4 - Jianhuang Li.

Author 5 - Shan Zeng.

Author 6 - Meizuo Zhong.

Author 7 - Lanhua Tang. 